\title{
Progress in Cue Exposure Therapy for the Treatment of Addictive Disorders: A Review Update
}

\author{
Temeia Martin, Steven D. LaRowe and Robert Malcolm*
}

Department of Psychiatry, Medical University of South Carolina, Charleston, SC 29425, USA

\begin{abstract}
Objectives: To evaluate the role of cue exposure therapy in addiction treatment.
Methods: A Pubmed/PsycInfo literature search was performed for cue exposure treatments for addictions from 2002 to 2009.

Findings: We located sixteen cue exposure therapy studies involving multiple addictions that were conducted between 2002 and 2009. Four of these studies consisted of clinical efficacy trials that assessed drug use after Cue Exposure Therapy (CET). In three of the four trials, cue exposure was no better or worse than the comparison therapy. Two trials showed somewhat worse retention in those receiving CET and one appeared to have higher relapse rates in those receiving CET. We did not find evidence of safety concerns in any of the four trials. Several promising methodological innovations included use of virtual reality, medication augmentation, investigation of renewal effects, use of fMRI methods, and study of individual differences.

Conclusions: Superior efficacy for CET over other forms of general treatment has not been demonstrated. However, research in this area may yet be advanced through adequately powered, multi-site CET trials combining multiple methodological innovations including investigation of individual differences and medication augmentation. Until such trials are conducted, cue exposure treatment for addiction will remain a speculative and uncommonly used therapy in clinical settings.
\end{abstract}

Keywords: Extinction, addiction, cue exposure therapy, review.

\section{INTRODUCTION}

Cue exposure therapy (CET) for the treatment of addictions is not widely used clinically and the effectiveness of CET remains a subject of debate [1]. This is in contrast to the widespread use of CET in the treatment of other psychiatric disorders. Exposure approaches to therapy have been shown to be effective treatment for anxiety disorders, including Post-Traumatic Stress Disorder [2] and specific phobias [3]. Despite encouraging early work [4], CET is not widely used in addiction treatment programs, though it continues to be the focus of a great deal of research. In a 2002 meta-analytic review, Conklin and Tiffany [1] examined the efficacy of cue exposure treatments for addiction across 18 treatment studies conducted between 1980 and 2002. While this earlier review concluded there was little evidence to support efficacy of cue exposure as part of addiction treatment, the authors identified a number of methodological weaknesses and provided suggestions for further research. The present review summarizes human cue exposure treatment trials for addictions published since 2002 , and examines the extent to which the state-of-thescience has improved within the past eight years.

*Address correspondence to this author at the Department of Psychiatry and Behavioral Sciences, Medical University of South Carolina, 67 President Street, MSC 861, Charleston, SC 29425, USA; Tel: (843) 792-5214; Fax: (843) 792-7353; E-mail: malcolmr@musc.edu

\section{BACKGROUND}

Pavlov's theory of classical conditioning [5] defined a learning process wherein an unconditioned stimulus (UCS) that elicits an unconditioned response (UCR) is consistently paired with a neutral stimulus until such time that the neutral stimulus itself can evoke a conditioned response similar to the unconditioned response. At that point, the neutral stimulus is designated the conditioned stimulus (CS) and the response it produces is designated as the conditioned response (CR). The process of extinction occurs when the CS is repeatedly presented without the accompanying UCS. The result is a weakening of the CR.

Individuals with substance abuse disorders are believed to undergo classical conditioning in their acquisition of addictive behaviors [1]. In this instance, the drug of abuse is the US and physiological and pharmacological responses induced by the drug comprise the UCR. As drugs and alcohol are used, previously neutral stimuli within the environment (context, sight/smell of the drug, items needed to use or ingest the drug) presumably become conditioned stimuli capable of inducing drug-relevant conditioned physiological responses. It is presumed that these conditioned stimuli, typically referred to as drug "cues," serve to activate CRs essential to the maintenance of drugseeking behavior. It has also been postulated that repeated exposure to these drug-related cues in the absence of drug use should result in extinction of drug-related cue reactivity, thereby reducing drug-seeking behavior. In other words, exposure to drug cues should extinguish the drug-relevant 
CRs in addicts. Along these lines, CET systematically exposes addicts to drug related cues, usually within a clinical setting, and this leads to extinction of drug-related cue reactivity. It is assumed that extinction of cue reactivity in the treatment setting should, in turn, translate into reduced reactivity to cues in real-world settings, with the ultimate benefit consisting of a reduction in problematic drug use.

Despite the continued intuitive appeal of the CET approach, there is little evidence that CET is superior to other forms of substance abuse treatment. In their 2002 meta-analytic review, Conklin and Tiffany concluded that "there is no consistent evidence for the efficacy of cueexposure treatment as currently implemented" [1] (p. 155). However, they qualified this conclusion by pointing out that there are many processes that can interfere with extinction, and may have done so in the CET interventions performed to date. These processes, referred by Conklin and Tiffany as "threats to extinction," include but are not limited to a) renewal (return of extinguished behavior within a novel context), b) spontaneous recovery, c) reinstatement (return of extinguished behavior in the presence of the UCS), and d) characteristics of the cues themselves (e.g. saliency). The authors suggest that CET may prove to be more efficacious if these so-called threats are methodologically ameliorated.

In the present review, we examined the extent to which recent CET studies have built upon the extant body of work since 2002. We examined whether studies had an index/control group, whether participants were inpatients or outpatients (allowing for control of substance use between CET sessions), the duration of follow-up post-treatment, the types of outcome measures, and dropout rates during treatment. Where possible, we highlight whether special care was made to address the aforementioned "threats to extinction." Perhaps most importantly, we qualitatively examined whether outcomes have improved given the present state of the science.

\section{METHOD OF REVIEW}

After a search of PubMed and PsychInfo databases, 16 studies were located that were published since 2002 and not included in Tiffany and Conklin's 2002 meta-analysis. Several studies published within the past eight years have consisted of attempts to improve CET methodology, but were not treatment studies designed to reduce drug use in real-world settings. Some were "proof-of-concept" investigations that examined the extent to which varied methods (e.g., video cue presentations, virtual reality presentations) evoked and/or reduced craving and physiological indicators of reactivity. Other studies systematically investigated the "threats to extinction" raised by Conklin and Tiffany [1], most notably the "renewal" effect. Finally, four were efficacy trials that examined the clinical impact of CET. Table 1 summarizes the results of these studies.

\section{Studies Examining Methods to Induce and Reduce Reactivity}

Bernaldo de Quirós Aragón et al. [6] attempted to develop a procedure to assess the impact of CET on classically conditioned responses to cues. Their cue reactivity assessment involved presenting five 90 -second videotaped drug-related scenes, with one 90 -second erotic scene serving as a control. Twenty-four outpatient detoxified opiate addicts were evaluated for level of craving, physiological reactivity (skin temperature, skin conductance, and heart rate), and positive/negative affect. Participants were assessed for reactivity to these scenes at pre-treatment, post-treatment, and six month follow-up. Twelve patients were assigned to a treatment as usual control condition. The remaining 12 were assigned to a CET intervention (six CET sessions per week for four weeks) and received treatment as usual. CET sessions were progressively intensive, initially consisting of presentation of drug paraphernalia (e.g., tinfoil, lighters, bag of drugs) and progressing to more complex and intense experiences (e.g., induction of emotional states, performing drug using rituals, in vivo exposure to drug using areas). By six months, nine participants had relapsed to heroin (three in the CET group, six in the control condition) and were excluded from the final assessment. The difference in relapse rate between conditions was non-significant. Results revealed that the video cues produced unexpectedly low levels of craving throughout the procedures, and significant reductions in craving were therefore not observed, though there was a trend that favored the CET group. In contrast, skin conductance reactivity was observed in both groups, with the CET group showing reduced skin conductance reactivity at six-month follow-up. Some differences were noted for negative and positive affect immediately post treatment, and findings favored the CET group. It is difficult to determine whether these modest effects were a result of the CET procedure itself, or a the result of a general effect of receiving additional time in treatment, as the CET intervention constituted an additional 14 hours of treatment relative to those in the treatment as usual condition.

Havermans and colleagues [7] compared CET to relaxation therapy (RT) in 70 inpatients undergoing drug and/or alcohol treatment. Patients were dependent on a variety of drugs (alcohol, cocaine, opiates, cannabis, benzodiazepines, or stimulants). Participants received 11 one-hour sessions, consisting of CET or RT. Immediately before the first and after the final session of the experimental interventions, participants were presented with personalized drug-related cues and a neutral cue (bar of soap) and assessed for reactivity (craving, skin conductance, finger pulse, and skin temperature). CET sessions consisted of two initial sessions that provided the treatment rationale and identified patient-relevant drug cues, followed by nine sessions of exposure to cues and imagery related to drug use. Attrition in this study was high, with a $44 \%$ drop out rate within the CET condition and $34 \%$ within the RT condition. Attrition generally resulted from relapse to drug use, followed by mandatory discharge from the treatment facility. Results failed to demonstrate that CET was more effective than RT, as cue-elicited subjective responses were reduced in both groups over the course of treatment. While urges declined over the sessions, skin conductance and heart rate did not decrease for either therapy. In contrast, peripheral vasoconstriction was reduced more effectively by relaxation therapy. Patients were not followed after discharge from the inpatient facility, so it is not possible to determine if CET provided any long-term clinical benefit. 
Table 1. Summary

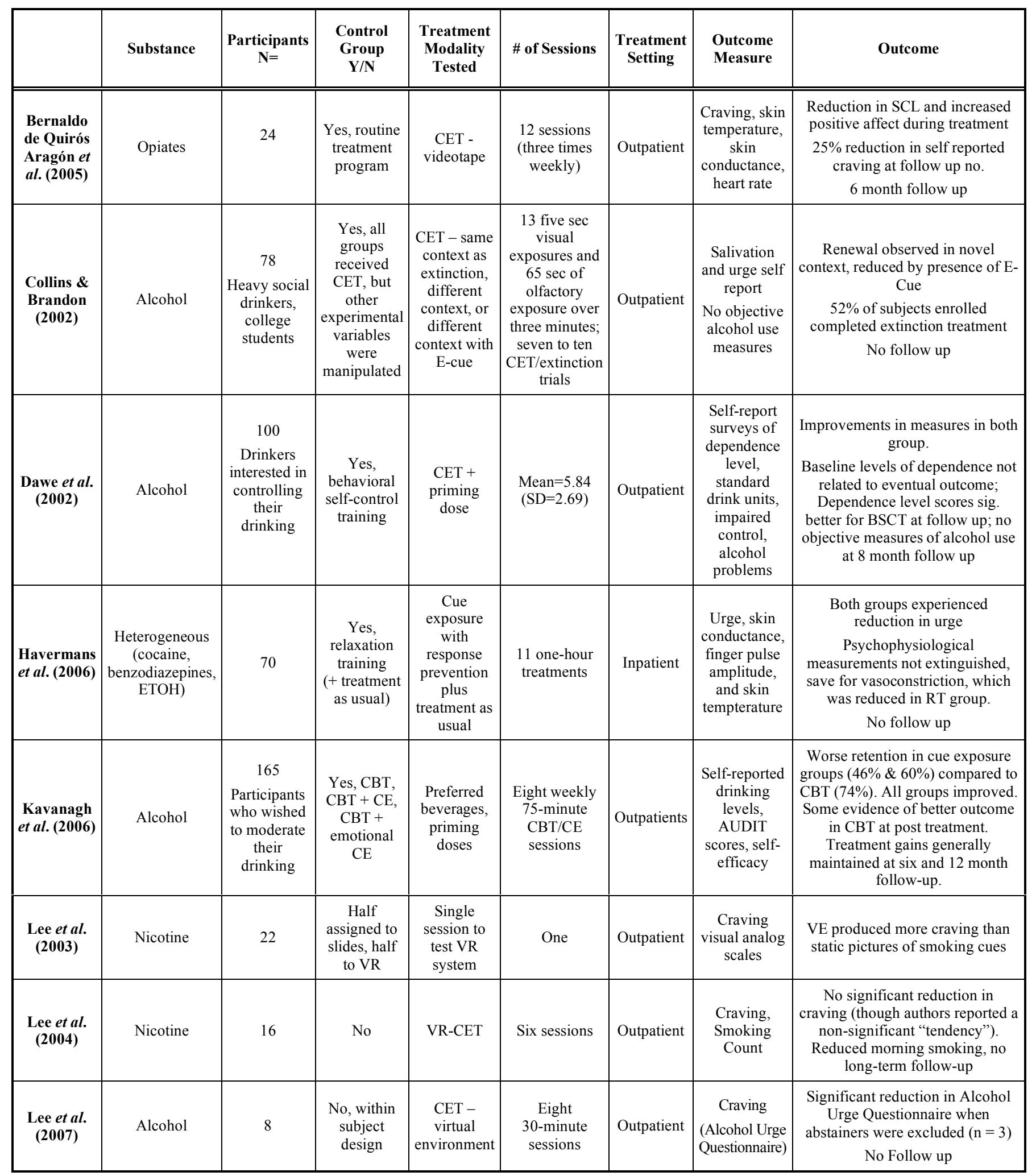




\begin{tabular}{|c|c|c|c|c|c|c|c|c|}
\hline & Substance & $\begin{array}{c}\text { Participants } \\
\mathrm{N}=\end{array}$ & $\begin{array}{l}\text { Control } \\
\text { Group } \\
\text { Y/N }\end{array}$ & $\begin{array}{l}\text { Treatment } \\
\text { Modality } \\
\text { Tested }\end{array}$ & $\begin{array}{c}\text { \# of } \\
\text { Sessions }\end{array}$ & $\begin{array}{c}\text { Treatment } \\
\text { Setting }\end{array}$ & Outcome Measure & Outcome \\
\hline $\begin{array}{l}\text { Loeber } e t \\
\text { al. (2006) }\end{array}$ & Alcohol & 63 & $\begin{array}{l}\text { Yes, CET } \\
\text { compared to } \\
\text { CBT }\end{array}$ & CET & $\begin{array}{c}\text { Nine 90- } \\
\text { minute } \\
\text { sessions } \\
\text { over three } \\
\text { weeks }\end{array}$ & Inpatient & $\begin{array}{l}\text { Drinking behavior assessed } \\
\text { by time-line follow back, } \\
\text { confirmed by objective and } \\
\text { corroborative reports; } \\
\text { Ratings of craving, self- } \\
\text { efficacy. Monthly follow- } \\
\text { ups to } 6 \text { months. In } \\
\text { addition, self ratings of } \\
\text { treatment practicality, and } \\
\text { treatment usefulness; }\end{array}$ & $\begin{array}{l}\text { More self-efficacy reported in } \\
\text { CET group. Patients found } \\
\text { CET content more practical } \\
\text { and useful than CBT. Both } \\
\text { treatments showed } \\
\text { improvements including } \\
\text { reduction in craving, alcohol } \\
\text { use, comparable increases in } \\
\text { days abstinence. Those with } \\
\text { higher dependence who } \\
\text { received CET showed more } \\
\text { days abstinent, less alcohol } \\
\text { consumed at the four to six } \\
\text { month follow-up. }\end{array}$ \\
\hline $\begin{array}{l}\text { Marissen } \\
\text { et al. } \\
\text { (2007) }\end{array}$ & Opiates & 127 & $\begin{array}{l}\text { Yes, placebo } \\
\text { psychotherapy } \\
\text { (relaxation } \\
\text { techniques } \\
\text { and emotion } \\
\text { management) }\end{array}$ & CET & $\begin{array}{l}\text { Nine one- } \\
\text { hour } \\
\text { session }\end{array}$ & Inpatient & $\begin{array}{l}\text { Drug-related cue reactivity } \\
\text { (skin conductance level, } \\
\text { urge self-report) }\end{array}$ & $\begin{array}{c}\text { Decreased self-reported urge in } \\
\text { both groups. } \\
\text { Decrease in skin conductance } \\
\text { in CET } \\
\text { Higher drop-out rates in CET } \\
\text { Follow-up at three months } \\
\text { indicated higher relapse in the } \\
\text { CET group }(40 \%) \text { compared to } \\
\text { the control group }(12 \%)\end{array}$ \\
\hline $\begin{array}{c}\text { Moon \& } \\
\text { Lee (2009) }\end{array}$ & Nicotine & 8 & $\begin{array}{l}\text { No, within } \\
\text { subject design }\end{array}$ & VR-CET & $\begin{array}{l}\text { Six } \\
\text { 20-minute } \\
\text { session }\end{array}$ & Outpatient & Functional MRI findings & $\begin{array}{l}\text { During VR-CET, smoking cues } \\
\text { produced greater activation in } \\
\text { PFC, L anterior cingulate, } \\
\text { R temporal and other cortical } \\
\text { areas relative to neutral cues. } \\
\text { Many of these areas remained } \\
\text { activated after six VR exposure } \\
\text { sessions, but two areas in the } \\
\text { PFC (superior and inferior } \\
\text { gyrii) showed reduced } \\
\text { activation after VR-CET. No } \\
\text { significant decreases in self } \\
\text { reported craving. }\end{array}$ \\
\hline
\end{tabular}


(Table 1) contd....

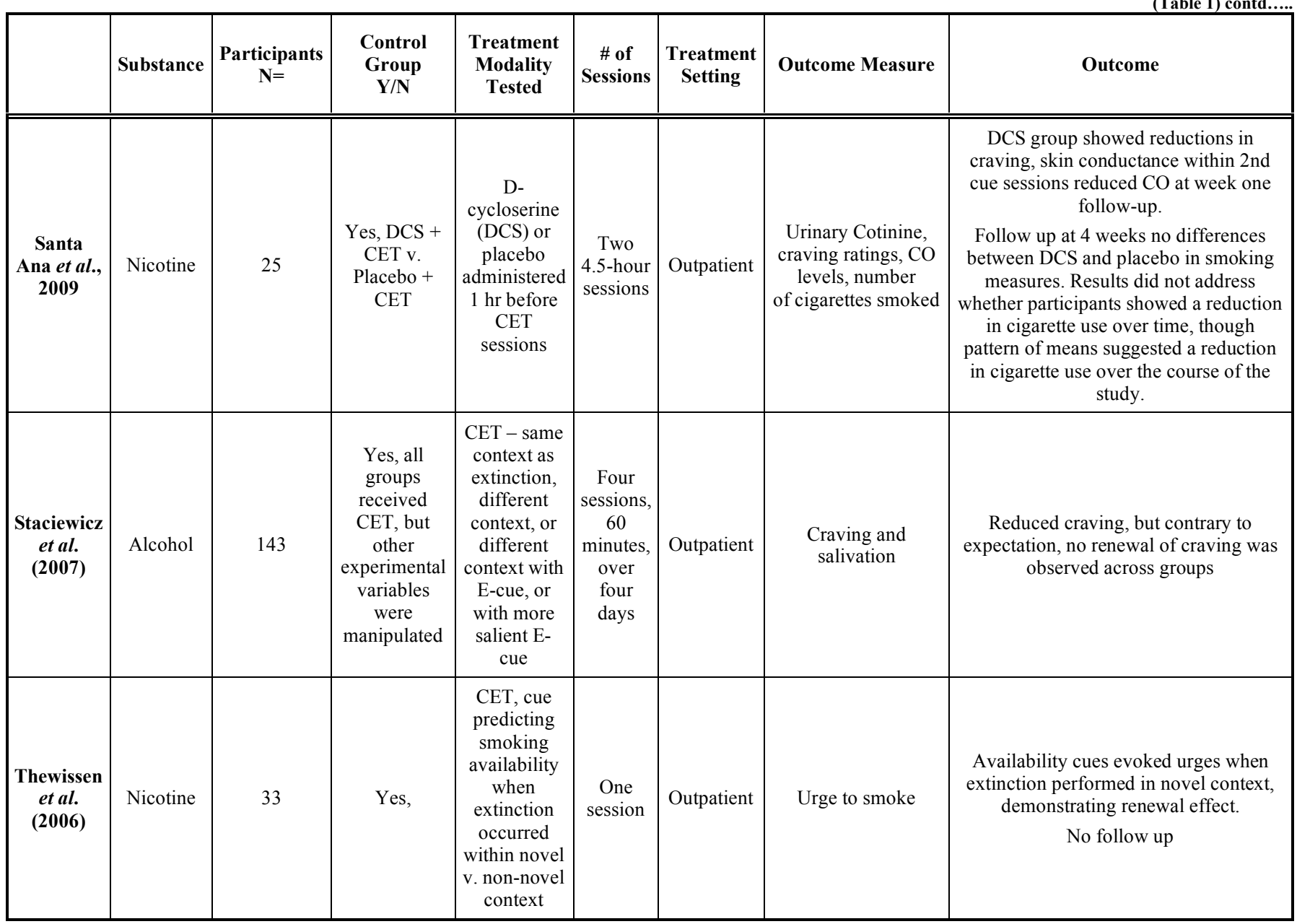

\section{Virtual Reality Studies}

Several published studies have attempted to advance CET methods by increasing the salience of cues using virtual reality (VR) technology. Lee et al. [8] compared the impact of VR and static pictures on craving levels. Twenty-two smokers were assigned to either the VR condition, consisting of a virtual smoking environment or static pictures; with results demonstrating that VR methods produced higher changes in craving than did static pictures. In a follow-up pilot study, these same researchers [9] examined whether this methodology would reduce craving in six late adolescent smokers. Over six sessions, participants spent up to 13 minutes in a VR smoking environment. Results suggested a trend for lower morning smoking levels across sessions, though no significant reduction in craving was noted. Lee and colleagues [10] evaluated the efficacy of virtual CET (VR-CET) in eight participants currently involved in Alcoholics Anonymous. Participants underwent 8 VR-CET sessions over four weeks. Cravings were measured using the Alcohol Urges Questionnaire. Results suggested that urges for alcohol measured during the final session were lower than those urges measured during the first.

Finally, Moon and Lee [11] examined the impact of VRCET on brain activation. Eight late adolescents underwent fMRI scanning prior to receiving VR-CET. During fMRI scans, participants were presented with photos of smoking cues and neutral objects. Participants then received six 20 minute sessions of VR-CET, after which a second scan was performed. Once again, the photographic cues were presented during the fMRI scan. Prior to VR-CET, smoking cues, relative to neutral cues, produced greater activation in the prefrontal cortex, the left anterior cingulate, and the right temporal lobe. While many of these areas showed continued activation to smoking related cues (relative to neutral cues), two areas in the prefrontal cortex (superior and inferior gyrii) showed reduced activation after VR-CET. There were no significant decreases in self reported craving, though there was some evidence that participants reduced their morning smoking levels over the course of the six sessions

In summary, it appears that cues presented in VR environment are more salient than static picture cues [8]. The evidence in VR studies reviewed here did not confirm that VR can lead to extinction of drug-related cue-induced craving. This may have been due to a failure to control for between session use of drugs (i.e., nicotine), providing an opportunity for participants to reacquire drug-cue pairings and essentially "reinstate" cue-elicited craving [1]. Moreover, while these studies were not specifically designed as clinical efficacy trials, the relative lack of long-term drug use data and lack of experimental control groups make it 
difficult to determine if VR interventions will translate to meaningful differences in actual clinical outcomes.

\section{Pharmacological Approaches to Cue Exposure Therapy and Extinction}

An innovative addition to extinction treatment studies has been pharmacologic augmentation. McClernon and colleagues [12] studied cue reactivity and cue extinction in 20 treatmentseeking smokers with blood oxygenation level dependent (BOLD) fMRIs. The first fMRI occurred after participants were allowed to freely smoke their regular brand of cigarettes for two weeks. While being scanned, subjects were shown pictures of smoking behaviors and neutral cues. Treatment included four weeks of reduced nicotine cigarettes (RNC), $21 \mathrm{mg}$ nicotine patches, and a guide to prepare for smoking cessation. The nicotine patches reduced withdrawal symptoms, while RNCs presumably made it possible to dissociate smoking behaviors from nicotine reward. After four weeks, subjects stopped the RNCs, and a second fMRI scan was performed. Participants then continued to use nicotine patches for four more weeks, received their final fMRI scan, and were then tapered off the patches in two final weeks. Results showed evidence of reduction of activation in response to smoking cues, relative to neutral, in the amygdala, an area believed to be involved in drug conditioning and extinction [13]. There was a modest reduction in salivary cotinine and breath carbon monoxide levels. In addition, four smokers achieved four weeks of continuous abstinence. Follow-up analyses examined whether there were differences at baseline between the four abstainers and remaining participants with respect to fRMI activation. Results indicated that the abstainers showed more thalamic activation at baseline when presented with smoking cues (relative to neutral cues), but showed reduced thalamic activation after using the RNCs and patches. In contrast, the remaining participants showed comparable thalamic activation to both cue types at all fMRI assessments. No other baseline characteristics differentiated the abstainers from the remaining participants. While the study suggested that reactivity to cues was reduced, as indicated by reduced activity in the amygdala, no control group was included, so it was not possible to unambiguously attribute this reduction to the procedures employed (i.e. RNCs and patches). On the other hand, the fact that differences in thalamic activation at baseline were associated with continuous (i.e. four-week) abstinence raises the possibility that individual differences in brain activation may interact with CET approaches. It may be that those most reactive to cues are more likely to show benefit from CET treatments.

D-cycloserine (DCS) is a partial N-methyl-D-aspartate (NMDA) agonist that has been shown to facilitate fear extinction in a number of studies $[14,15]$. Postulating that D-cycloserine would facilitate CET, Santa Ana and associates [16] studied 25 treatment-seeking smokers in a randomized controlled trial of CET plus DCS or placebo. All participants had two, 4.5-hour CET sessions two weeks apart. Participants were required to have overnight abstinence from smoking prior to each session and four hours following each session. Placebo or fifty milligrams of DCS was given orally one hour before CET. At one week follow-up, the DCS group had a significantly lower Carbon Monoxide $(\mathrm{CO})$ level $(p<0.01)$. There was no significant difference in $\mathrm{CO}$ levels at week four. Skin conductance and self reported urges to smoke declined significantly from both pre- to post-sessions for the DCS group. Although this study used CET methodology, it set out to compare CET + DCS to $\mathrm{CET}+$ Placebo, and therefore provides little additional information about the effectiveness of CET $v s$ other forms of treatment. On the other hand, these results suggest that CET may be enhanced with proper medication augmentation. This suggests that CET may indeed prove to be superior to other forms of treatment if used in conjunction with these medications.

\section{Studies Investigating the "Renewal" Effect}

As noted earlier, the review by Conklin and Tiffany suggested that previous CET work may have suffered from effects of renewal. Renewal occurs when a conditional stimulus (CS) is paired with an unconditioned stimulus (UCS) in one context (context A). Extinction (i.e., exposure) then occurs in a new context (context B). After extinction training, there is a return to the original context (context A), which leads to a renewal of responding to the CS. Conklin and Tiffany argue that many CET treatments are essentially conducted in a novel context (context B, e.g., the therapy room) relative to the context in which CS and US pairings originally occur (context A, i.e., the original drug-using environment). These authors suggest that some ways of circumventing the problem of renewal, such as having cue exposure occur in multiple contexts, including "extinction reminders" (i.e., portable cues that can be with the patient during treatment and when they return to their original context), and/or increasing the inter-trial intervals between extinction sessions. At present, there are a number of studies that have attempted to incorporate these suggestions into the research and implementation of CET procedures.

Collins and Brandon [17] conducted a methodologicallyoriented study that explicitly examined the extent to which changes in context increased the likelihood of renewal of cue reactivity in 78 social drinkers. Participants underwent a baseline assessment during which they were initially exposed to an alcoholic beverage (i.e., beer). Assessments of urges and saliva levels were collected as participants viewed, handled, and sniffed the alcoholic beverage. After this baseline assessment, participants underwent a minimum of seven exposure trials (approximately three minutes each) within a single 30-50 minute session. Participants who did not meet initial extinction criteria (i.e., return to baseline urge) received as many as 10 trials. After completion of the cue exposure trials, participants engaged in a distracting activity for 25 minutes. After this, they were again presented with cues and assessed for any return or "renewal" of urges. While one third of the participants underwent all assessments and cue exposure in the same room (i.e., all procedures occurred in context A), two thirds of the participants received cue exposure in a room that was different from the room in which the initial and renewal assessment occurred (i.e., CET trials occurred in context B).

Results indicated that that participants who received all assessments and cue exposure within the same context showed reduced levels of reactivity during the final cue reactivity assessment. In contrast, those individuals who received cue exposure sessions in the novel context showed a return (i.e., "renewal") of reactivity within the final cue reactivity assessment. These results suggested that receiving extinction training in a novel context followed by a return to 
one's original context may indeed lead to renewal of previously extinguished reactivity.

It should be noted, however, that these researchers also demonstrated that this renewal effect can be moderated. Consistent with the suggestion of Conklin and Tiffany regarding the inclusion of an extinction reminder, all participants received cue exposure in the presence of an extinction cue consisting of a brightly colored clipboard and pencil. The extinction cue was present in the final testing for half of those participants who had received cue exposure in the novel context. The results showed that the effect of renewal was reduced in the presence of the extinction cue. Taken together, the results suggest that renewal is likely to occur when cue exposure is performed in a novel context (as usually occurs in CET), but this effect may be moderated if an appropriate extinction cue is present during cue exposure as well as when the patient returns to the original context.

In an attempt to replicate and extend the findings of Brandon and Collins, Staciewicz, and colleagues [18] also tested whether conducting cue exposure trials in a novel context would result in renewal and whether an extinction reminder would moderate this finding. In addition, however, these investigators assessed whether the extinction reminder would be more effective if the saliency of the extinction reminder was increased. Another innovation was an attempt to increase the amount of time between extinction sessions by having those sessions occur over four consecutive days (in contrast to Collins and Brandon, who conducted extinction sessions on a single day). One-hundred forty-three alcohol dependent outpatient subjects participated. Subjects attended four sessions over four consecutive days for 60 minutes each. Exposure trials consisted of handling and sniffing the participant's preferred alcoholic beverage. After exposure, during which craving level was extinguished, renewal was tested under four possible conditions: within the original exposure/extinction context, within a novel context with no extinction cue, a novel context containing the extinction cue, or a novel context with the extinction cue with enhanced saliency. It was hypothesized that following extinction, participants who received all procedures in the same context would show continued reduction in alcohol urges; those who received exposure in the novel context would show renewal, with this effect moderated by the presence of the extinction cue. The results of the study did not support the hypotheses; no group differences were observed at the final testing period. Indeed, no renewal was observed in any group, as all groups showed a reduction in craving overall.

Thewissen and colleagues [19] evaluated renewal of urge to smoke in acquisition and extinction contexts. This experiment went "back to basics" by actually pairing smoking behavior with specific cues consisting of two serving trays, blue or yellow in color. Participants were told that one of the trays would signal that smoking would occur while the other indicated smoking would not occur. Patients were then evaluated for craving in the presence of these cues. Next, patients were escorted into a room where they were allowed to take a puff of a cigarette on three trials in the presence of the serving tray that signaled smoking, but only handled cigarettes (without smoking them) on three more trials in the presence of the tray that signaled no smoking. This "acquisition phase" lasted about 30 minutes. Next, patients were moved to a new room (novel context) to undergo an "extinction" (i.e. cue exposure) phase in which they completed identical procedures as in the "acquisition phase," but without being allowed to smoke in the presence of either tray. Finally, half of the participants remained in the room in which they underwent extinction training, while half were returned to the original room in which they underwent acquisition training. This manipulation was intended to test if a return to the original acquisition context would once again lead to greater craving in response to the availability cue, i.e., lead to a "renewal" effect.

Results indicated that the acquisition manipulation was effective, as the tray that signaled smoking produced greater craving than that which signaled no smoking. Extinction of craving via cue exposure was effective as well, as the smoking cue became less effective in evoking craving by the end of extinction training. Return to the original context produced renewal, as the smoking cue was once again able to produce a greater level of craving than the unavailability cue. In contrast, those participants that remained in the same context throughout the entire experiment showed little or no differentiation in cravings in the presence of either cue. This study provided another demonstration that effects of extinction can essentially be reversed if the original acquisition context differs substantially from that in which extinction training occurs.

MacKillop \& Lisman [20] also evaluated renewal and the effects of undergoing extinction in varied contexts. They studied 73 heavy alcoholics who underwent three sessions of extinction followed by a renewal session. Sessions occurred over four consecutive days. The sessions lasted 40 minutes with assessment of urge and salivation at 10 minute intervals. Participants were assessed for renewal in a novel context after receiving cue exposure within a single context (three sessions over three days in the same context), multiple contexts (three sessions over three days in three different contexts), and a neutral control (three sessions over three days in three different contexts, but with neutral cues). It was hypothesized that those who received cue exposure in the same context would show the greatest renewal in the novel context, while those who received cue exposure in multiple contexts would show attenuated levels of renewal. The findings, however, showed that those who were exposed to cues in the single context showed no evidence of renewal, and in fact showed the greatest levels of extinguished cue reactivity. No benefit was noted for having received extinction training in multiple contexts.

The above described studies represent a response to the earlier suggestion by Conklin and Tiffany to assess "threats to extinction" by assessing 1) the extent to which renewal occurs, and 2) means to offset the renewal effect. Two of these studies demonstrated that renewal does appear to occur when extinction/cue exposure is conducted in a context that is novel relative to the context in which drug-cue pairings are acquired. While the renewal effect was not observed in all studies, there appears to be sufficient evidence that it can indeed occur. Thus, if future trials are to be implemented effectively, the potential impact of renewal should be considered and addressed in future experimental designs.

\section{Recent Efficacy Trials}

There have been only a handful of studies in the past eight years that have conducted bona fide CET efficacy trials (i.e., assessed clinical efficacy, performed long-term followups). In one of these studies, Dawe and colleagues [21] 
compared a cue exposure intervention to a behavioral self control treatment (BSCT) designed to teach controlleddrinking skills. One hundred participants were recruited on the basis of their desire to cut down or control drinking. Participants in the CET condition underwent eight exposure sessions following standard CET procedures [22]. CET involved presentation of a priming dose of the participant's preferred drink, followed by a hierarchy of exposure to drinking cues that involved a progression of cue presentation starting with looking at the drink, then handling it, and then smelling it. Participants were assessed for urges every four minutes and had to provide urge ratings within $10 \%$ of baseline ratings before proceeding to the next step in the hierarchy.

In addition to the standard CET intervention, this study incorporated a number of methodological improvements over previous CET studies. First, the authors attempted to address the confound of renewal by requiring participants to complete homework assignments in which they exposed themselves to cues in a variety of real-world contexts. Secondly, the researchers took care to present the cues in manner similar to that in which alcohol is typically consumed (e.g., champagne in a champagne glass). Finally, participants were presented with an actual dose of alcohol at the beginning of each extinction session to provide extinction training for interoceptive cues. Despite these improvements, follow-up data at eight months revealed that there were no clinically meaningful differences between the two groups (though both improved), save for a reduction in rated dependence that favored the BSCT group. However, some limitations in the CET approach were noted, including a lack of response prevention training. In addition, participants were able to drink between sessions (consistent with the "controlled drinking" approach). This may have allowed for reacquisition of cue-drug pairings during the course of the CET interventions possibly leading to reinstatement of cue-related reactivity and a reduction of treatment efficacy.

Kavanagh et al. [23] conducted a large, multi-site trial that examined the efficacy of CET in participants with alcohol abuse who report drinking when dysphoric. The study compared cognitive behavioral therapy (CBT) to combined interventions of CBT plus cue exposure (CE) and CBT plus CE after a dysphoric mood induction (the emotional cue exposure or ECE condition). The study was aimed at improving controlled drinking levels. Participants received eight weekly outpatient sessions for 75 minutes each. The participants in the $\mathrm{CE}$ condition received $\mathrm{CE}$ beginning the second session, during which they resisted drinking when exposed to alcohol cues along with priming doses of their preferred beverage. In the third session, those in the ECE condition recalled negative experiences prior to $\mathrm{CE}$, so that they could undergo cue exposure procedures in the context of negative emotions. Results indicated that the CBT condition had a $74 \%$ retention rate. The cue exposure condition had a retention rate of $60 \%$, while the emotional cue exposure group had the worst retention of $46 \%$. While all groups improved with respect to self-reported consumption immediately post-treatment, the results suggested that those in the CBT group fared somewhat better, contrary to expectations. Lowered drinking levels across the three groups were maintained at six and 12 months. It should be noted that $\mathrm{CE}$ sessions were designed to have participants "resist" the urge to drink, but they could drink if they chose to. It should also be noted that if drinks were consumed, this occurred during "discussion of the CBT material" (p. 1109). Thus, the authors acknowledged that "attention and memory for the material may also have been affected by concurrent consumption of alcohol during part of the CBT."

In a 2006 study, Loeber and colleagues [24] compared CET to CBT delivered in nine 90-minute sessions over a 3week period in 63 inpatients receiving detoxification from alcohol. Measures of craving, self-efficacy, and drinking behavior were assessed monthly up to six months after treatment. This study collected additional ratings of perceived usefulness and practicality of the treatment approaches. In general, patients felt CET was more "practical" and "useful" than CBT, though patients receiving either treatment showed improvements in craving and drinking measures during the follow-up period. In contrast, patients receiving CET reported higher ratings of selfefficacy. Moreover, patients with greater levels of dependence who received CET tended to show better outcomes with respect to days abstinent and amount of alcohol consumed within the four to six month follow-up period.

Marrisen and colleagues [25] performed a traditional CET study in opiate addiction. One-hundred twenty seven inpatient participants undergoing treatment as usual in a drug-free therapeutic community treatment were randomized to CET or a control condition consisting of placebo psychotherapy (relaxation techniques and emotion management). Treatments were given after two-weeks of detoxification over a three-week period. Patients were followed for three months after treatment. Participants were assessed for cue reactivity (self reports of urge and mood, along with measures of skin conductance level) during a cue reactivity assessment session that involved presenting a video cue depicting drug use, combined with an olfactory cue consisting of vaporized heroin. CET procedures involved presentation of highly individualized drug cues presented across nine one-hour sessions. Persons receiving placebo psychotherapy completed nine one-hour sessions of comparable length. Results indicated that both groups showed comparable reduction in craving to heroin cues over the course of treatment. Those in the CET group showed a reduction in skin conductance in response to cues. Regarding clinical outcomes, more drop out was observed at three months in the CET (50.8\%) compared to the placebo psychotherapy group $(22.6 \%)$. In addition, a greater percentage $(40.0 \%)$ of those in the CET relapsed compared to the placebo psychotherapy group $(12.9 \%)$ based on selfreports of use.

\section{SUMMARY}

Sixteen studies relevant to CET published since 2002 were identified. Of these studies, the majority were methodological in nature and only four were randomized clinical trials meeting criteria of randomization, comparison therapy, and outcomes related to substance use [21, 23-25]. Four methodological studies used virtual reality technology [811]. Initial evidence suggests that virtual cues may be substantially more salient than static picture cues [8], though 
even among these studies a significant reduction in craving after cue exposure was not always observed [9, 11]. Two studies suggested that individual differences may influence response to CET. One study [24] found that individual differences in alcohol dependence was related with CET outcomes, with those having higher levels of dependence showing better outcomes after receiving CET. Another study of individual differences using fMRI methods [12] found that smokers who eventually achieved abstinence showed greater levels of baseline thalamic activation in response to smoking cues (relative to neutral) as well as a reduction of thalamic activation after CET procedures. These results suggest that regional brain activity may be a prognostic marker of individual response to CET interventions.

A number of the recent CET studies made explicit efforts to study and/or control the effects of renewal, one of the "threats to extinction" identified by Conklin and Tiffany [1]. Two studies $[17,19]$ were able to demonstrate renewal effects under experimental conditions. In addition, one of these two studies [17] showed that renewal effects could be reduced if participants had a portable extinction cue with them during cue exposure and during the period in which renewal occurred. Among the clinical efficacy trials reviewed, at least one [21] attempted to address the issue of renewal by having participants practice CET procedures at home as part of a homework assignment, though individuals in the CET group did not fare better than the comparison control group with respects to clinical outcome. Taken together, while later studies failed to replicate earlier positive findings regarding renewal effects $[18$, 19], or failed to find enhanced efficacy of CET when taking renewal into account [21], the body of research in this area remains small. Thus, there is arguably enough positive evidence to merit further systematic investigation of this phenomenon.

While several studies set out to systematically study and/or address the potential effects of renewal within CET trials, none set out to examine the impact of reinstatement, which is another potentially important "threat to extinction" identified by Conklin and Tiffany. Reinstatement is process wherein previously extinguished cue reactivity reappears after return to drug use. Theoretically, use of drugs or alcohol between exposure sessions should lead to reinstatement, thus weakening the impact of CET. In general, the studies reviewed here did not make efforts to control for drug use between CET sessions. There were three inpatients studies [7,24, 25], however, and it is reasonable to assume that participants in these studies did not have easy access to drugs. This arguably should have protected against reinstatement. Despite this added control, only one of the three [24] showed any superior efficacy for CET, while another showed worse outcome [25], and still another failed to find evidence of reduced physiological reactivity (i.e., skin conductance level). Thus, at present, there is not enough data to determine whether controlling for in-between session use enhances the efficacy of CET interventions.

With respect to craving reduction, the evidence suggests that individuals receiving CET achieve this aim $[10,16,17,19]$ though some only showed a "tendency" to achieve this $[6,9]$, and at least one study failed to show any such craving reduction [11]. Importantly, it is not clear that CET itself leads to reduction of craving, or if this reduction merely reflects habituation to the novel experimental context or the effects of participation in treatment in general. Indeed, within at least two of the clinical trials, there were comparable reductions in craving within both the CET and comparison conditions [24, 25].

In three clinical trials involving alcohol abuse, the efficacy of CET was no worse or better than comparison treatments. Dawe et al. [21] showed that while those receiving CET showed improvements at eight months, CET was not superior to the comparison treatment (Behavioral Self Control Therapy). Similarly, Loeber et al. [24] found that those receiving CET showed improvements in outcome, but again those improvements were comparable to those observed within the comparison therapy group (Cognitive Behavioral Therapy). In the third study involving alcohol-dependent patients, Kavanagh [23] found evidence of improvement in the both the CET and comparison groups. In contrast, while CET fared as well as (but not better than) comparison therapy in the studies involving alcohol abuse and dependence, the controlled trial involving opiate addicts showed evidence of worse outcome in those receiving CET, as indicated by higher dropout rates during the study and greater relapse rates at three month follow up [25].

When evaluating the quality of the evidence for CET efficacy, one must consider dropout rates, follow-up assessments, and incorporation of objective assessments of drug use. Among the studies reviewed, dropout rates were sometimes difficult to determine. Those that compared dropout rates between CET and control interventions seemed to suggest more attrition in groups receiving CET. Kavanagh et al. [23] had a dropout rate ranging from 40 to $54 \%$ in groups receiving CET, but only $26 \%$ in those receiving CBT alone. Havermans and colleagues [7] had a dropout rate of $44 \%$ for the CET group but only $34 \%$ for the relaxation therapy group. Marissen and colleagues [25] had a 15\% dropout rate for the CET group and a dropout rate of $5 \%$ for the relaxation therapy group. In studies that did not include a comparison group, rates of dropout remained high. Collins and Brandon [17] reported a $48 \%$ dropout rate. McClernon and colleagues [12] reported a $25 \%$ dropout rate. Nine of 24 participants (37.5\%) dropped out of the study by Bernaldo de Quirós Aragón and colleagues [6]. Aside from the clinically relevant aspects of dropout, poor retention levels also may weaken the ability to interpret and generalize the empirical findings. Therefore, future work should strive to 1) increase retention, and 2) incorporate novel statistical methods that can more effectively address the impact of missing data.

\section{CONCLUSION}

Consistent with the previous review by Conklin and Tiffany [1], there continues to be little evidence for the superior efficacy of CET over other forms of substance abuse treatment. However, it should be emphasized that the efficacy trials did not find CET to be ineffective; indeed CET subjects improved significantly from baseline, though these improvements did not differ from the other active treatment conditions. There have been innovations in the literature, however, that have yet to be incorporated into clinical trials. These innovations include the use of VR technology, attention to potentially important individual differences, systematic studies of "threats to extinction," and the use of pharmacological approaches to enhance the impact of CET. Studies investigating CET continue to be challenged by a number of methodological problems, including small sample sizes, high dropout rates, lack of objective measures of substance use and lack of procedures for 
preventing substance use between extinction sessions. In order adequately address these issues, a multi-site trial with adequate statistical power that simultaneously addresses individual differences, includes medication augmentation, and attempts to neutralize threats to extinction may be needed to determine whether there is any incremental benefit of including CET in substance abuse treatments, or whether CET should be "put on the shelf" for good.

\section{ABBREVIATIONS}

$$
\begin{array}{ll}
\text { BOLD } & =\text { Blood Oxygenation Level Dependent } \\
\text { BSCT } & =\text { Behavioral Self Control Training } \\
\mathrm{CBT} & =\text { Cognitive Behavioral Therapy } \\
\mathrm{CE} & =\text { Cue Exposure } \\
\mathrm{CET} & =\text { Cue Exposure Therapy } \\
\mathrm{CO} & =\text { Carbon Monoxide } \\
\mathrm{CR} & =\text { Conditioned Response } \\
\mathrm{CS} & =\text { Conditioned Stimulus } \\
\mathrm{DCS} & =\text { D-cycloserine } \\
\mathrm{ECE} & =\text { Emotional Cue Exposure } \\
\mathrm{fMRI} & =\text { Functional Magnetic Resonance Imaging } \\
\text { NMDA } & =\text { N-methyl-D-aspartic acid } \\
\text { RNC } & =\text { Reduced Nicotine Cigarettes } \\
\text { RT } & =\text { Relaxation Therapy } \\
\text { UCR } & =\text { Unconditioned Response } \\
\text { UCS } & =\text { Unconditioned Stimulus } \\
\text { VR } & =\text { Virtual Reality } \\
\text { VR-CET } & =\text { Virtual Reality Cue Exposure Therapy } \\
\text { ACKNOWLEDGEMENTS }
\end{array}
$$

The authors would like to thank Matthew Smith, Lindsay Ayer, and Vanessa Milsom for their helpful comments and assistance in the revision of this manuscript.

\section{REFERENCES}

[1] Conklin CA, Tiffany ST. Applying extinction research and theory to cue-exposure addiction treatments. Addiction 2002; 97: 155-67.

[2] Nemeroff C, Dremner J, Foa E, Mayberg H, North C, Stein M. Posttraumatic stress disorder: a state-of-the-science review. J Psychiatr Res 2006; 40: 1-21.

[3] Wolitzky-Taylor K, Horowitz J, Powers M, Telch M. Psychological approaches in the treatment of specific phobias: a meta-analysis. Clin Psychol Rev 2008; 28: 1021-37.

[4] Childress AR, McLellan AT, O'Brien CP. Abstinent opiate abusers exhibit conditioned craving, conditioned withdrawal and reductions in both through extinction. Br J Addict 1986; 81:655-60.

[5] Pavlov IP. Conditioned Reflexes: an investigation of the physiological activity of the cerebral cortex 1927; London: Oxford University Press, pp. 43-77.
[6] Bernaldo de Quirós Aragón M, Labrador FJ, de Arce F. Evaluation of a group cue-exposure treatment for opiate addicts. Span J Psychol 2005; 8:229-37.

[7] Havermans RC, Mulkens S, Nederkoorn C, Jansen A. The efficacy of cue exposure with response prevention: extinguishing drug and alcohol cue reactivity. Behav Intervent 2006; 22: 121-35.

[8] Lee JH, Ku J, Kim K, et al. Experimental application of virtual reality for nicotine craving through cue exposure. CyberPsychol Behav 2003; 6: 275-80.

[9] Lee JH, Lim Y, Graham SJ, et al. Nicotine craving and cue exposure therapy by using virtual environments. CyberPsychol Behav 2004; 7 : 705-13.

[10] Lee JH, Kwon H, Choi J, Yang BH. Cue-exposure therapy to decrease alcohol craving in virtual environment. CyberPsychol Behav 2007; 10 617-23.

[11] Moon J, Lee JH. Cue exposure treatment in a virtual environment to reduce nicotine craving: a functional MRI study. CyberPsychol Behav 2009; 12: 43-5.

[12] McClernon FJ, Hiott FB, Liu J, Salley AN, Behm FM, Rose JE. Selectively reduced responses to smoking cues in amygdala following extinction-based smoking cessation: results of a preliminary functional magnetic resonance imaging study. Addict Biol 2007; 12: 503-12.

[13] See RE, Fuchs RA, Ledford CC, McLaughlin J. Drug addiction, relapse, and the amygdala. Ann N Y Acad Sci 2003; 985: 294-307.

[14] Ressler KJ, Rothbaum BO, Tannenbaum L, et al. Cognitive enhancers as adjuncts to psychotherapy: use of D-cycloserine in phobic individuals to facilitate extinction of fear. Arch Gen Psychiatry 2004; 61: 1136-44.

[15] Wilhelm S, Buhlmann U, Tolin DF, et al. Augmentation of behavior therapy with D-cycloserine for obsessive-compulsive disorder. Am J Psychiatry 2008; 165: 335-41.

[16] Santa Ana EJ, Rounsaville BJ, Frankforter TL, et al. d-Cycloserine attenuates reactivity to smoking cues in nicotine dependent smokers: A pilot investigation. Drug Alcohol Depend 2009; 104: 220-7.

[17] Collins BN, Brandon TH. Effects of extinction context and retrieval cues on alcohol cue reactivity among nonalcoholic drinkers. J Consult Clin Psychol 2002; 70: 390-7.

[18] Staciewicz PR, Brandon TH, Bradizza CM. Effects of extinction context and retrieval cues on renewal of alcohol-cue reactivity among alcohol-dependent outpatients. Psychol Addict Behav 2007; 21: 244-8.

[19] Thewissen R, Snijders SJBD, Havermans RC, Van Den Hout MA, Jansen A. Renewal of cue-elicited urge to smoke: implications for cue exposure treatment. Behav Res Ther 2006; 44: 1441-9.

[20] MacKillop J, Lisman, SA. Effects of a context shift and multiple context extinction on reactivity to alcohol cues. Exp Clin Psychopharmacol 2008; 16: 322-31

[21] Dawe S, Rees VW, Mattick RP, Sitharthan T, Heather N. Efficacy of moderation-oriented cue exposure for problem drinkers: a randomized controlled trial. J Consult Clin Psychol 2002; 70: 1045-50.

[22] Drummond DC, Glautier S. A controlled trial of cue exposure in alcohol dependence. J Consult Clin Psychol 2004; 62: 809-17.

[23] Kavanagh DJ, Sitharthan G, Young RM, et al. Addition of cue exposure to cognitive-behaviour therapy for alcohol misuse: a randomized trial with dysphoric drinkers. Addiction 2006; 101: 11061116.

[24] Loeber S, Croissant B, Heinz A, Mann K, Flor H. Cue exposure in the treatment of alcohol dependence: effects on drinking outcome, craving and self-efficacy. Br J Clin Psychol 2006; 45: 515-29.

[25] Marissen MA, Franken IH, Blanken P, Van den Brink W, Hendriks VM. Cue exposure therapy for the treatment of opiate addiction: results of a randomized controlled clinical trial. Psychother Psychosom 2007; 76: $97-105$. 